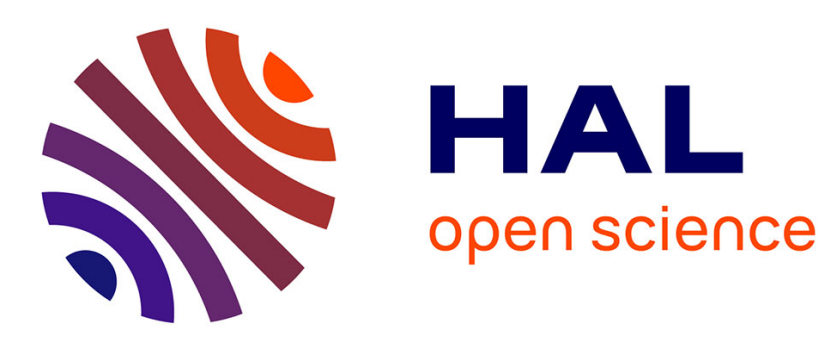

\title{
Postulat de la monnaie et théorie de la valeur chez Marx
} Nicolas Piluso

\section{To cite this version:}

Nicolas Piluso. Postulat de la monnaie et théorie de la valeur chez Marx. Revue de la régulation. Capitalisme, institutions, pouvoirs, 2014, 15, pp.1-10. hal-01070165

\section{HAL Id: hal-01070165 https://hal.science/hal-01070165}

Submitted on 30 Sep 2014

HAL is a multi-disciplinary open access archive for the deposit and dissemination of scientific research documents, whether they are published or not. The documents may come from teaching and research institutions in France or abroad, or from public or private research centers.
L'archive ouverte pluridisciplinaire HAL, est destinée au dépôt et à la diffusion de documents scientifiques de niveau recherche, publiés ou non, émanant des établissements d'enseignement et de recherche français ou étrangers, des laboratoires publics ou privés. 


\title{
Postulat de la monnaie et théorie de la valeur chez Marx Postulate of money and value in Marx's theory
}

\author{
PUBLIE DANS LA REVUE DE LA REGULATION, 15 | 1er semestre /Spring 2014
}

\author{
Nicolas Piluso \\ Maître de conférences, CERTOP (UMR CNRS 5044), Université Paul Sabatier-Toulouse III, \\ nicolas.piluso@iut-tlse3.fr
}

\begin{abstract}
L'article tente de réévaluer la portée de la critique de Carlo Benetti au sujet de la théorie de la monnaie marxienne. Si effectivement il n'est pas possible de déduire logiquement la théorie de la monnaie de la théorie de la marchandise chez Marx, nous proposons de postuler la monnaie à l'intérieur même de la théorie de la valeur. Ce type de postulat serait fondamentalement différent du postulat keynésien de la monnaie qui conduit à rejeter la théorie de la valeur et de la répartition. Cette proposition de "postulat marxien de la monnaie » présente l'avantage de conserver les enseignements qu'apporte l'analyse marxienne de l'économie monétaire.
\end{abstract}

The paper attempts to reevaluate the criticism of Carlo Benetti about the marxian theory of money. If indeed it is impossible to deduce the existence of money from value theory, we propose to postulate money within the theory of value. This type of assumption is fundamentally different from the keynesian postulate of money which leads to reject the theory of value and distribution. This proposition of a "marxian postulate of money" has the advantage of preserving the teachings brought by the marxian analysis of the monetary economy.

Mots clé : marchandise, monnaie, valeur, Etat, crise économique

Key words: commodity, money, value, State, economic crisis

\section{Introduction}

L'approche du postulat de la monnaie dont Keynes (1971), dans le Treatise on Money, apparaît comme un défenseur, conduit à expurger toute idée de théorie de la valeur. Postuler l'existence de la monnaie revient à affirmer que le langage de l'économie relève non pas de la nature et de choix individuels mais d'une création politique qui se cristallise dans la monnaie. Une économie sans monnaie n'est alors pas concevable, aussi bien d'un point de vue historique que théorique, ce qui revient à rejeter tout à la fois la problématique de la neutralité (la monnaie a-t-elle une influence sur l'économie réelle ?) et de l'essentialité (l'équilibre avec monnaie est-il préférable que l'équilibre sans monnaie ?).

Cartelier $(1996,2007)$ réhabilite aujourd'hui le postulat de la monnaie de Keynes sur la base du constat que la théorie de la valeur néoclassique et la théorie des décisions individuelles sont incompatibles avec l'introduction de la monnaie dans l'analyse économique.

La théorie de la régulation a quant à elle suivi une démarche semblable à celle de Cartelier en rejetant la théorie de la valeur et en adoptant une analyse d'emblée monétaire de 
l'économie. C'est ce que souligne Montalban (2012) : «La plupart des membres de la théorie de la régulation, notamment Aglietta et Orléan (2002) [...] se sont distingués en critiquant tout recours à une théorie de la valeur, en lui substituant une théorie de la monnaie et en s'intéressant plutôt aux fondements sociaux de la monnaie. Cette position se fonde sur l'idée d'un choix nécessaire entre théorie de la monnaie et théorie de la valeur ».

Cependant, la question des rapports entre théorie de la valeur et postulat de la monnaie apparaît plus complexe chez Marx, car pour ce dernier, l'économie capitaliste dont il souhaite rendre compte est une économie monétaire ${ }^{1}$ alors même que la base de cette économie monétaire est la marchandise : Marx (1977) annonce dès le chapitre 1 du livre 1 du Capital que le fondement de la théorie du capital et de la monnaie est la théorie de la marchandise. La théorie marxienne souffrirait-elle donc d'une contradiction sur ce point ? Autrement dit, est-il possible de concevoir l'économie dans sa dimension monétaire en adoptant la théorie de valeur de Marx ? Telle est la problématique centrale des développements qui suivent.

D'après la critique de Benetti (1985), il serait impossible de déduire la monnaie de la théorie de la valeur, ou, de façon plus rigoureuse, d'intégrer la monnaie à la théorie des marchandises, quel que soit le champ analytique considéré (celui des néoclassiques comme celui de Marx). En conséquence, il conviendrait d'abandonner la théorie marxienne des formes de la valeur au même titre que la théorie walrasienne.

Cette critique de la théorie marxienne de la monnaie, doublée d'une remise en cause de la théorie de la valeur (Benetti, Cartelier, 1999), a constitué le point de départ d'un abandon de l'approche marxiste pour lui substituer une approche directement monétaire de l'économie. Le postulat de l'unité de compte a donné lieu à des travaux sur le fonctionnement des économies monétaires dans la droite lignée de la pensée keynésienne (Cartelier et Frydman, 2001 ; Cartelier, 2007 ; Bauvert, 2003 ; Julien, 2001). Au sein de la théorie de la régulation, l'approche directement monétaire conduit les économistes à considérer que «valeur et monnaie constituent une seule et même réalité » (Lordon et Orléan, 2007).

En raison du rejet radical de la théorie de la valeur et de la monnaie marxienne et donc de la production d'une théorie des prix alternative, Montalban (2012) pointe le risque de dérive de la théorie de la régulation vers la théorie néoclassique. Nous souhaitons donc ouvrir un débat en nous demandant s'il n'est pas possible de concevoir un postulat de la monnaie différent de celui proposé par Keynes et Benetti-Cartelier, et de revenir ainsi aux fondements marxistes de la théorie de la régulation telle qu'exposée par Aglietta en 1976. Il sera envisagé dans le présent article que ce postulat soit posé à l'intérieur même du champ analytique de la théorie de la valeur marxienne. Le but est de pouvoir conserver chez Marx une approche monétaire de l'économie et ses apports théoriques précieux pour l'analyse économique ${ }^{2}$. Cela permettrait en outre de protéger la théorie de la régulation de la dérive possible soulignée plus haut (Montalban, 2012). L'ambition de l'article n'est pas de régler tous les problèmes liés à la théorie monétaire marxienne, mais de faire des propositions théoriques nouvelles ouvrant l'espace à un débat au sein de la théorie de la régulation et de la théorie marxiste.

Dans un premier temps, nous présentons l'analyse marxienne de la monnaie et la critique nanterroise qui lui est adressée. Dans un second temps, nous proposons une autre lecture de la genèse de la monnaie fondée sur l'énoncé de ce qui pourrait être appelé le " postulat marxien de la monnaie ».

\footnotetext{
${ }^{1}$ D'ailleurs, Aglietta (1976) adopte la théorie de la monnaie de Marx dans ses premiers travaux.

2 Segura (1995) a montré dans la revue l'Actualité économique que la théorie de la monnaie marxienne permet de combler les lacunes de la théorie keynésienne du circuit.
} 


\section{L'analyse de Marx et la critique française}

La genèse de la monnaie renvoie à la théorie de la marchandise et de la valeur dont le point d'aboutissement est la monnaie ; la première des fonctions de la monnaie, mesure des valeurs, est le point d'aboutissement de l'évolution des formes de la valeur.

Après avoir étudié la valeur, Marx passe à l'analyse de ses formes de manifestation. Lorsque deux marchandises s'échangent, ces dernières jouent chacune un rôle indissociable l'un de l'autre mais opposé. La première marchandise (que l'on appelle A) exprime sa valeur au travers d'une seconde $(\mathrm{B})$. « $\mathrm{A}$ » est la forme relative, « $\mathrm{B}$ » la forme équivalente, c'est-àdire que « $\mathrm{A}$ » exprime sa valeur relativement à une autre marchandise, qui est son miroir de valeur. Dans l'expression « $\mathrm{A} »=\langle\mathrm{B} »$, le signe «égal » signifie non seulement identité qualitative, en tant que formes phénoménales d'une même substance, la valeur, mais aussi égalité quantitative, en tant que cristallisation d'une même grandeur de valeur. Elle stipule que « $\mathrm{A}$ » exprime sa valeur grâce à la rencontre de « $\mathrm{B}$ ». Il existe donc un sens de lecture de cette égalité et en tant que membre de gauche, " $\mathrm{A}$ » ne peut jouer le rôle de « $\mathrm{B}$ » et vice versa. Cependant, si la valeur de «A » peut-être exprimée par « B », elle peut l'être tout aussi bien par les marchandises $« \mathrm{Z} », 《 \mathrm{~V} »$ ou « $\mathrm{Y} »$. Marx passe alors à l'analyse de la forme développée de la valeur.

Dans cette dernière forme, «A » exprime sa valeur au travers d'une multitude de marchandises: la valeur des marchandises revêt indifféremment toute forme de valeur d'usage (Marx, 1977). La grandeur de valeur de chacune des marchandises règle les proportions dans lesquelles elles s'échangent.

C'est l'inversion de la forme 2, nous dit Benetti (1985) qui est cruciale dans la tentative d'intégration de la monnaie à la théorie de la valeur.

Dans les formes simple et développée, les marchandises expriment leur valeur comme quelque chose de distinct de leur valeur d'usage. Dans la forme générale (ou forme monnaie, qui lui est équivalente), toutes les marchandises expriment leur valeur au travers d'un seul et unique équivalent et toute nouvelle espèce de marchandise entrant dans la sphère de circulation est contrainte de faire de même. L'équivalent est la forme officielle de la valeur, l'expression sociale du monde des marchandises (Marx, 1977). Pour qu'une marchandise s'isole et devienne équivalent général ou monnaie, il faut qu'elle soit rejetée du monde des marchandises. L'équivalent n'est plus une marchandise en ce sens qu'elle incorpore une valeur immédiatement sociale. Dépourvue de forme valeur relative, elle possède le monopole social de forme d'expression des valeurs.

Benetti rejette la théorie de la monnaie marxienne dans la mesure où à leurs yeux, cette tentative d'intégration de la théorie de la monnaie à la théorie de la valeur conduit à un échec. Exposons ce point de vue.

La forme développée de la valeur (F2) ne signifie pas qu'une marchandise exprime sa valeur relative en toutes les autres, et qu'elle possède donc (N-1) équivalents particuliers dans un monde à $\mathrm{N}$ marchandises. La caractéristique de cette forme $\mathrm{F} 2$ est précisément l'absence d'équivalent unique. En fait, il existe $\mathrm{N}(\mathrm{N}-1)$ expressions des valeurs relatives et donc la même quantité d'équivalents particuliers. Dans ce type d'organisation sociale, il n'existe donc pas de marchandises mais de simples produits ou valeurs d'usage. Cette absence d'équivalent général fait que les relations sociales ne peuvent être mesurées par des grandeurs, des prix, constitutives des relations économiques.

Dans la forme valeur générale, F3, les marchandises expriment leur valeur par rapport à une seule et unique marchandise. Il existe donc un équivalent général qui permet la formation des valeurs relatives. C'est seulement à partir de ce stade que les produits du travail deviennent marchandises. Or, d'où vient, d'après Marx, cette forme F3 ? Elle émane de l'inversion de F2. Or, l'inverse de F2 ne peut qu'engendrer sa propre image, c'est-à-dire F2. 
D'après Benetti, il n'est possible d'obtenir F3 par inversion de F2 que si l'on postule au stade de F2 l'unicité de la marchandise qui exprime sa valeur relative. «Or, une telle hypothèse est arbitraire et incompatible avec la forme F2 elle-même » (Benetti, 1985). Il n'est finalement pas possible de déduire la forme monnaie de la forme développée car l'inversion de la forme développée ne donne pour résultat qu'elle-même, c'est-à-dire la forme développée. Dans le même ordre d'idée, Deleplace (1985) parle de "rupture » entre F2 et F3 et le fait que la monnaie renferme une valeur validée d'emblée par la société empêche de considérer qu'elle émane du monde des marchandises.

Benetti en conclut radicalement, compte tenu de ce que F3 n'est pas le résultat de l'inversion de F2, que le lien entre monnaie et valeur doit être rejeté. Il rejoint ainsi Cartelier qui considère que l'opposition fondamentale entre les courants théoriques n'est finalement pas la théorie de la valeur, mais le choix entre analyse réelle (constitutive de la théorie de la valeur) et analyse monétaire (constitutive du " postulat de la monnaie »). La théorie de la valeur serait par construction incompatible avec une théorie de l'économie monétaire. Si la thèse selon laquelle Marx échoue à déduire logiquement la monnaie de la valeur peut être acceptée, il semble possible de d'adopter une autre alternative que celle du postulat de la monnaie tel que Cartelier et Benetti le proposent.

\section{Une autre lecture de la genèse de la monnaie}

Après un repositionnement des problèmes posés par la genèse de la monnaie, la piste d'une intégration du postulat de l'unité de compte (tel que Benetti et Cartelier le conçoivent) dans la théorie marxienne sera explorée. Cette possibilité ne résistant pas à l'analyse, une autre piste sera identifiée : celle d'un autre postulat de monnaie intégré à la théorie de la valeur. Un tel dépassement de la critique de Benetti et Cartelier permet de conserver un apport particulier de Marx à la théorie économique : le fondement monétaire de la crise.

\section{1. La monnaie chez Marx : les termes du problème posé}

Reformulons le problème tel que Marx le présente.

La monnaie procède de l'échange des marchandises, auquel on peut appliquer la théorie de la valeur travail (Foley, 2000 ; Bellofiore, 1989). Néanmoins, des éléments de la théorie marxienne de la monnaie laissent à penser que la monnaie n'est pas une marchandise incarnant un travail privé destiné à devenir travail social ; une marchandise est travail privé en tant que valeur d'usage, renfermant une certaine dépense de force de travail, et travail social en tant qu'objet échangé, représentante d'une fraction pertinente du travail global de la société. Or, pour Marx, la monnaie n'est représentante que d'un travail immédiatement social. Sa fonction d'équivalent lui confère le statut de marchandise d'emblée reconnue socialement : l'argent est la marchandise qui a pour caractère l'aliénabilité absolue (Marx, 1977). Si la monnaie est travail social, alors par définition il n'est jamais privé. La monnaie a ceci de particulier qu'elle n'a pas à être validée a posteriori par le marché. Elle n'est donc pas par définition une marchandise (Deleplace, 1985).

On trouve donc apparemment chez Marx une analyse duale de la monnaie. D'un côté, il y a une tentative d'intégrer la monnaie à la théorie de la valeur, et de l'autre, on trouve les formes d'une sorte de postulat de l'unité de compte semblable à celui de Keynes : Marx (1977) ne dit-il pas en effet que seul un acte social peut faire d'une marchandise déterminée un équivalent général, et que l'or-monnaie ne vaut comme tel que là où il a derrière lui le pouvoir de l'État? Cet acte social relèverait donc en fait pour Marx de l'État en tant que représentant de la société. Le postulat de l'unité de compte n'est rien moins que de conférer à la monnaie une dimension d'emblée collective (c'est l'État qui institue l'unité de compte). Pour Salama (1992), cela signifie en clair que « les rapports marchands ne peuvent être conçus simplement comme des rapports entre agents privés, en l'absence d'un rapport centralisé, étatique, qui 
institue l'équivalent général [...] C'est ce que Marx semble avoir en vue lorsqu'il indique que le pouvoir de l'équivalent général est coextensif de celui de l'État et que le monnayage est un monopole étatique ».

La pensée de Marx rejoindrait donc, sous certains aspects, la pensée de Keynes qui s'inspire elle-même de_Knapp (1973). Selon ce dernier, la monnaie est « une créature de la loi » car l'État choisit et instaure une forme monnaie qui s'impose aux individus sur un territoire donné. L'acceptation de la monnaie par les agents économiques est garantie par l'État. Mais le problème qui se pose du point de vue de la théorie marxienne de la valeur est que selon cette tradition keynésienne, la monnaie n'est pas nécessairement liée à la valeur d'une marchandise. Simmel (1999) souligne à cet égard que la monnaie devient, avec le développement des échanges, une quantité mesurable des marchandises échangées indépendamment de sa valeur marchande. Selon cet auteur, la monnaie engendrerait en outre un surcroît de valeur car sa spécificité serait d'exprimer « la totalité des buts ».

Si la monnaie apparaît comme le produit d'un acte social non marchand, comment faire le lien entre la monnaie et l'économie ? Est-il possible de concilier la théorie de la valeur de Marx et le postulat de la monnaie tel que Cartelier et Benetti le proposent ?

\section{2. L’incompatibilité entre postulat keynésien de la monnaie et théorie marxienne}

Deux arguments conduisent à abandonner l'idée selon laquelle le postulat keynésien de la monnaie et la théorie de l'analyse de Marx sont conciliables.

Tout d'abord, l'État n'est concevable dans le cadre de l'analyse marxienne que comme une instance dont le fondement est constitué par le rapport de domination entre classes sociales. L'État ne peut donc pas être pensé en analyse marxienne indépendamment des rapports de production qui fondent les rapports de domination. Or, dans la théorie de la valeur et de la monnaie, nulle référence n'est faite aux rapports de production capitalistes. C'est ce que de Brunhoff (1967) souligne : " Marx a commencé son étude de la production capitaliste par une analyse des marchandises, des échanges et de la circulation qui se réfère à la notion d'une production marchande vide de rapports sociaux déterminés : la monnaie apparaît sans ses déterminations capitalistes ». Et pour cause, la théorie de la marchandise et de la monnaie constituent le fondement de la théorie du capital, autrement dit des rapports de production capitalistes donc de la forme capitaliste des rapports de domination entre classes sociales. L'État ne peut donc pas avoir sa place dans une théorie de la monnaie acceptable dans un cadre marxien dépassant le seul discours économique. C'est pourquoi le postulat de la monnaie, au sens où c'est l'État qui est fondement de l'analyse économique, n'est pas compatible avec l'analyse marxienne. Si l'on veut mettre l'État au cœur du fait « monnaie », alors il faut abandonner l'idée de Marx que les concepts de valeur et de monnaie sont indispensables à la construction du concept de capital.

Par ailleurs, la monnaie est (selon Marx) cette marchandise exclue du monde des marchandises, c'est-à-dire de la circulation d'ensemble. L'or n'est-il pas monnaie que lorsqu'il est dépouillé de ses uniformes nationaux ?

Il n'y a donc pas de monnaie au sens marxien du terme tant que la sphère de circulation des marchandises n'est pas unifiée et elle ne peut s'unifier sans l'émergence d'un équivalent général. Il y existe un rapport dialectique entre l'expulsion d'une marchandise du monde des marchandises pour jouer ce rôle de monnaie et la constitution de ce monde (cette circulation unifiée des marchandises). La monnaie au sens marxien du terme nie l'État-nation comme autorité monétaire, expression d'un morcellement de la sphère de circulation des marchandises. Le capitalisme, qui a pour logique la production de marchandises sur une échelle croissante, pose la nécessité d'une interpénétration des sphères de leur circulation et, 
ce faisant, celle de l'émergence d'une monnaie. Marx s'éloigne donc de la tradition du « postulat de la monnaie ».

C'est fondamentalement ce qui justifie la distinction entre la forme 3 et la forme 4 de la valeur. Pourquoi distinguer forme générale et forme monétaire puisque substantiellement la monnaie n'est qu'un équivalent général ? Le concept d'équivalent général permet d'identifier les formes primitives de la monnaie : l'or sous forme d'espèces circulant pour leur valeur faciale et imposées par l'État comme telles alors qu'elles jouent, comme la monnaie achevée mais dans une sphère restreinte, le rôle de mesure des valeurs, de moyen de circulation et de réserve de valeur. Au niveau du processus historique, la forme générale correspond à l'expression du besoin de monnaie, c'est-à-dire d'unification de la sphère de circulation des marchandises. La forme monétaire correspond à la phase (qui n'existe pas encore) marquée par l'existence de la sphère unifiée.

Face à une telle incompatibilité entre le postulat keynésien de la monnaie et l'approche de Marx, nous proposons de déplacer la position et la nature du postulat de la monnaie dans l'enchaînement logique qui amène à l'analyse de l'échange marchand.

\section{3. Vers une proposition de postulat marxien de la monnaie}

Chez Keynes, « les relations économiques ne s'édifient pas sur une table rase [...] mais s'inscrivent dans un certain contexte institutionnel»(Cartelier, 1996). L'État institue et définit la valeur de la monnaie. Il ne s'agit donc plus de fonder l'analyse économique sur une liste d'individus et de biens relevant de la nature, mais sur des relations économiques d'emblée institutionnalisées.

Chez Marx, le point de départ de son analyse est la marchandise, car il considère que cette dernière est le présupposé de la monnaie, qui elle-même est le présupposé du capital. Comme chez les économistes néoclassiques, on retrouve dans l'analyse marxienne l'émergence de la problématique monétaire dans le constat de l'impossibilité de l'échange marchand dans une économie de troc.

Soit en effet une économie à 3 agents (I, II et III) et à 3 biens (A, B, C). I possède A et désire du $\mathrm{B}$; II possède $\mathrm{B}$ et désire du $\mathrm{C}$; III possède $\mathrm{C}$ et désire du $\mathrm{A}$. L'échange ne pourra pas avoir lieu en raison de ce que Clower (1969) appelle l'absence de double coïncidence des besoins.

La monnaie intermédiaire dans les échanges ayant été évacuée de l'analyse néoclassique de Debreu, la solution au problème vient de l'hypothèse de la maison de compensation, autrement dit de la centralisation des échanges.

Dans le cadre marxien, la solution au problème est une marchandise jouant le rôle d'intermédiaire ; mais elle ne peut jouer ce rôle que si elle est l'équivalent général : les deux fonctions sont logiquement et indissolublement liées sur la base de la théorie de la valeur (Segura, 1974). C'est ce que signifie le passage de F2 à F3 : la poursuite du développement des échanges suppose qu'une marchandise joue le rôle d'intermédiaire donc d'équivalent général. L'équivalent-général-intermédiaire émerge comme une nécessité de l'extension des échanges.

S'il n'est pas logiquement possible de déduire la monnaie de la valeur, il est possible en revanche de poser « un postulat marxien de la monnaie » à ce stade de l'analyse (passage de F2 à F3) : il est postulé ici que ce sont les individus (et non l'État) qui, finalement, considérés collectivement choisissent dans le monde des marchandises la monnaie officielle. La monnaie est acceptée par tous car tous croient que les autres l'acceptent. L'idée sous-jacente est celle du mécanisme de boostrap que l'on retrouve dans les modèles de prospection. C'est ce qu'Iwaï (1996) souligne en affirmant que « money is money because it is used as money » : le choix de la monnaie s'explique par lui-même, au sens où un individu utilise la monnaie parce qu'il croit que les autres font de même. 
Ainsi, il est possible de supposer que la monnaie est engendrée au sein de groupes sociaux n'appartenant pas à une entité coiffée par un "État », une instance ayant la capacité d'imposer un équivalent général. On peut considérer des cité-États au sein desquelles l'échange n'existe pas mais qui commercent entre elles; dans ce commerce, une marchandise joue le rôle d'équivalent-intermédiaire qui n'est imposé par aucune instance mais procède d'un consensus. Le corps social sélectionne une marchandise particulière qui devient monnaie.

Rappelons le concept de la monnaie renvoie au monde des marchandises, sans segmentation territoriale. Le corps social est l'ensemble de tous ceux qui se rapportent les uns aux autres comme propriétaires des produits candidats à l'échange. Si ce concept de corps social devait renvoyer à l'État, ce serait un État dont le pouvoir s'étendrait sur ce monde. Or, cela n'est que difficilement concevable, à moins de refondre les concepts marxiens d'État et de monnaie.

Sur les rapports entre corps social, État et monnaie, de Brunhoff (1967) confirme notre interprétation de Marx en soulignant que même au sein d'un espace national, le pouvoir monétaire de l'État "n'implique pas une capacité de déterminer la valeur de la monnaie » (p. 61). Le pouvoir monétaire de l'État est subordonné « à la détermination de la forme monnaie simple telle qu'elle a été exposée plus haut. Le rapport social qui fonde l'existence et le rôle de la monnaie est celui de l'échange privé entre producteurs de marchandises » (souligné par nous).

Il s'agit donc ici de reformuler le propos de la genèse de la monnaie : cette dernière conduirait à postuler que la monnaie est un produit sélectionné par la collectivité pour devenir l'équivalent général. Cela revient à affirmer que la monnaie ne vient pas de nulle part (c'est-àdire que pour Marx, la marchandise-monnaie n'est monnaie que parce qu'elle est d'abord marchandise) : c'est une marchandise dont la valeur est réglée par la quantité de travail abstrait; en tant qu'elle possède le caractère d'aliénabilité absolue néanmoins, elle est exclue du monde des marchandises.

Ainsi, il faut distinguer, pour appréhender la monnaie, la genèse de son statut. Du point de vue de son fondement, une marchandise devient monnaie que parce qu'elle a préalablement été une marchandise. Du point de vue de son statut, la monnaie n'est pas une marchandise dans la mesure où elle est le produit d'un travail immédiatement social.

\section{4. Les conséquences analytiques du postulat marxien de la monnaie}

Le fait que les économistes néoclassiques échouent à intégrer la monnaie dans leur théorie de la valeur justifie la démarche de Benetti et Cartelier d'abandonner le recours à la problématique de la valeur. Dans le modèle d'équilibre général d'Arrow Debreu (qui est à horizon fini), il a été démontré que la monnaie n'est pas intégrable dans les fonctions d'utilité puisque le prix d'équilibre de la monnaie est nul (Hellwig, 1993). Si la théorie de la valeur n'est pas invalidée pour autant, il n'en demeure pas moins que l'absence totale de monnaie dans l'analyse est problématique puisqu'elle interdit de penser le déroulement des échanges marchands (Cartelier, 1996).

Il en va de même chez Marx dès lors qu'on considère qu'il échoue à déduire la monnaie de la valeur.

Cet échec analytique rend parfaitement intelligible la proposition de Benetti et Cartelier de réhabiliter la démarche keynésienne consistant à faire table rase de la théorie de la valeur pour penser d'une manière différente le fonctionnement de l'économie (Cartelier, 1996).

Si l'on postule la monnaie au sein de la théorie néoclassique standard, cela ne conduit pas à modifier en profondeur l'analyse positive de l'économie étant donné le résultat de neutralité (Patinkin, 1956). C'est ce que montre en substance la théorie quantitative de la monnaie, en 
vertu de laquelle la masse monétaire en circulation n'affecte pas l'économie réelle (niveau de production, niveau d'emploi, et structure des prix relatifs des marchandises).

$\mathrm{Si}$ en revanche, le postulat marxien de la monnaie est accepté, il est possible de conserver un «acquis» de l'analyse marxienne : celui du fondement monétaire de la possibilité de crise $^{3}$.

Segura (1974) a en effet souligné le fait que chez Marx, la possibilité de crise est logiquement fondée sur la théorie de la monnaie. Marx propose donc sur la crise un cadre d'analyse théorique différent de celui proposé par les tenants du postulat keynésien de la monnaie. Rappelons brièvement le raisonnement qui permet d'aboutir à ce résultat.

La monnaie est d'abord une marchandise choisie par le corps social pour exprimer la valeur des autres marchandises : elle est mesure des valeurs. Mais cette fonction de mesure des valeurs n'a de sens que si les marchandises s'échangent; c'est ainsi que la monnaie permet également la circulation des marchandises : "la forme prix renferme en elle-même l'aliénabilité des marchandises et la nécessité de cette aliénation » (Marx, 1977, p. 113). L'échange n'étant que la conversion de la monnaie en marchandises, la circulation générale de ces dernières appellent une certaine quantité de monnaie: "la quantité de monnaie qu'exige la circulation de toutes les marchandises présentes au marché est donc déterminée par la somme totale de leurs prix. La monnaie ne fait que représenter réellement cette somme d'or déjà exprimée idéalement dans la somme des prix des marchandises. L'égalité des deux sommes se comprend donc d'elle-même " (Marx, 1977, p. 124). Or, rien ne peut assurer $a$ priori que la masse monétaire corresponde parfaitement aux besoins de la circulation. Si la masse de monnaie est excédentaire, il est logiquement nécessaire de concevoir que la monnaie puisse être thésaurisée. Segura (1991) montre ainsi avec Marx que la fonction "moyen de thésaurisation » découle logiquement des deux premières fonctions de la monnaie sur la base de la théorie de la valeur.

Mais le problème posé par la thésaurisation est qu'elle n'a ni règle ni mesure. Il en résulte que la quantité de monnaie effectivement thésaurisée peut être plus grande que celle nécessaire. Autrement dit, la nature monétaire de l'économie fonde donc la possibilité de mévente, c'est-à-dire la possibilité de crise de surproduction. C'est cette idée que Marx met en évidence lorsqu'il écrit que la monnaie scinde l'identité de la vente et de l'achat en deux actes distincts : " Si la séparation des deux actes complémentaires l'une de l'autre de la métamorphose des marchandises se prolonge, si la scission entre la vente et l'achat s'accentue, leur liaison intime s'affirme par une crise » (Marx, 1977, p. 121) ${ }^{4}$.

Ainsi, l'utilité de «conserver» l'analyse monétaire de Marx est qu'elle impacte la théorie de la valeur elle-même : toute marchandise ne renferme pas nécessairement une valeur. À compter du moment où elle ne trouve pas d'équivalent pour l'exprimer, ou, autrement dit, dès lors qu'elle ne rencontre pas effectivement la monnaie pour s'échanger, « le travail dépensé inutilement ne crée pas de valeur » (Marx, 1977, p. 56).

\section{Conclusion}

L'objet de cet article a été de fournir une grille de lecture alternative à celle de l'école française nanterroise à propos de la théorie marxienne de la monnaie. D'après cette dernière, le postulat de la monnaie doit se substituer à la théorie de la valeur comme fondement de l'analyse économique. Marx essuie certes un échec en tentant de prolonger sa théorie de la valeur par une théorie de la monnaie. La thèse défendue ici est qu'il est cependant possible de déplacer la nature et la position du postulat keynésien de la monnaie au sein même de

\footnotetext{
${ }^{3}$ Il est évident que le maintien du couple théorie de la valeur/théorie de la monnaie chez Marx permet aussi $a$ priori de conserver son analyse du capital et de la plus-value.

${ }^{4}$ Pour qu'une telle possibilité de crise se mue en nécessité, il convient d'enrichir l'analyse et d'y introduire le concept de capital.
} 
l'analyse de Marx. Sa nature est modifiée car ce n'est plus l'État qui institue de la monnaie mais la collectivité. Sa position est également changée car ce n'est plus le point de départ de l'analyse, mais la clé du passage de la forme F2 à la forme F3 de la valeur.

Ainsi, il reste possible d'affirmer avec Marx que la marchandise n'a pas une manière d'être conforme à son concept avant que la monnaie ait elle-même une manière d'être conforme à son concept, c'est-à-dire avant que le capital n'existe et soit parvenu à un certain degré de développement. Dire cela, c'est dire que le concept préexiste à ses formes de manifestation et que la réalité n'est pas appréhendée comme la théorie qui en rend compte. Le concept de marchandise permet de penser celui de monnaie, si le " postulat marxien " est accepté ; ensemble, ils permettent de penser le concept de capital. Dans la réalité, c'est le capital qui conditionne l'existence du marché du monde dans le cadre duquel la monnaie et donc la marchandise acquièrent une existence conforme à leur concept ${ }^{5}$.

Le postulat de l'unité de compte adopté par l'école nanterroise affirme la dimension arbitraire que renferme la monnaie. De la même manière, Marx pourrait affirmer l'arbitraire que représente le choix collectif d'une monnaie dans le monde des marchandises et par là même le passage de F2 à F3. Marx peut ainsi fournir un principe explicatif à la valeur de grâce à la base analytique que lui fournit la théorie de la valeur (Segura, 1995); de même, il peut proposer une analyse de la crise de l'économie monétaire sur une base logique différente de celle des keynésiens. Enfin, la théorie de la régulation, en réintroduisant l'analyse marxienne de la valeur et de la monnaie (comme le suggère Harribey, 2011), serait immunisée contre la possibilité de dérive néoclassique en matière de théorie des prix.

Aglietta M. (1976), Régulation et crises du capitalisme, Paris, Calmann-Lévy.

Aglietta M. et Orléan A. (2002), La monnaie entre violence et confiance, Paris, Odile Jacob.

Bauvert J. (2003), «L'ambivalence du concept de liquidité dans le Treatise On Money », L'actualité économique, vol. 79, $\mathrm{n}^{\circ} 1-2$, p. 87-100.

Bellofiore R. (1989), “A monetary Labor Theory of Value”, Review of Radical Political Economics, ${ }^{\circ} 21$, p. 1-25.

Benetti C. (1985), «Économie monétaire et économie de troc : la question de l'unité de compte commune », Économie appliquée, $\mathrm{n}^{\circ} 1$.

Benetti C. (1997), «Monnaie, choix individuels et frictions », journée d'études «John Hicks », Paris Panthéon Sorbonne.

Benetti C., Cartelier J. (1999), "Market and division of labour: a Critical reformulation of Marx's view”, Rivista di Politica Economica, vol. 89, n 4-5, p. 117-139.

Boseley F. (2001), A critique of Benetti's critique of Marx's derivation of the necessity of money, working paper, Mount Holyoke College, Massachussetts, USA.

Brunhoff S. de (1967), La monnaie chez Marx, Éditions sociales.

Cartelier J. (1985), «Théorie de la valeur ou hétérodoxie monétaire : les termes d'un choix », Économie appliquée, $\mathrm{n}^{\circ} 1$.

Cartelier J. (1996), La monnaie, Dominos Flammarion.

Cartelier J. (2001), «Monnaie et marché : un point de vue critique sur les modèles de prospection », Revue économique, $\mathrm{n}^{\circ}$ 5, p. 993-1011.

Cartelier J. (2007), "The Hypostasis of Money: An Economic Point of View", Cambridge Journal of Economics, vol. 31, Issue 2, p. 217-233.

Cartelier J., Frydman R (2001), L'économie hors de l'équilibre, Paris, Economica.

${ }^{5}$ C'est ce qui a amené Marx à affirmer ceci : «C'est dans le commerce entre nations que la valeur des marchandises se réalise universellement [...] C'est sur le marché du monde, et là seulement, que la monnaie fonctionne dans toute la force du terme [...] Sa manière d'être y devient adéquate à son idée » (Marx, 1977, p. 147). 
Clower R.W. (1969), Monetary Theory Introduction, Penguin Books.

Deleplace G. (1985), «Sur quelques difficultés de la théorie de la monnaie marchandise Chez Ricardo et Marx », Économie appliquée, $\mathrm{n}^{\circ} 1$.

Foley D.K. (2000), "Recent Developments in the Labor Theory of Value", Review of Radical Political Economics, March, p. 1-39.

Hahn F.H. (1965), "On some problems of providing the Existence of an Equilibrium in a monetary economy", in The theory of interest rate, F.H. Hahn and F.P.R. Brechling (eds.), London, Macmillan.

Harribey J.-M. (2011), «André Orléan, L'empire de la valeur, Refonder l'économie, Paris, Seuil, $2011 \Perp$, Revue de la régulation, $\mathrm{n}^{\circ} 10,2^{\mathrm{e}}$ semestre.

Hellwig M. (1993), "The challenge of money theory", European Economic Review, n 37, p. 215-242.

Iwaï K. (1996), "The Boostrap theory of money: a search theory foundation of Monetary Economics", Structural change and Economic Dynamic, ${ }^{\circ}$ 7, p. 451-477.

Julien L. (2001), "Échanges effectifs et chômage keynésien ", in L'économie hors de l'équilibre, J. Cartelier, R. Frydman (dir.), Paris, Economica.

Keynes J.M. (1971) [1930], Treatise on Money, tomes V et VI in Collected Writtings of John Maynard Keynes, Royal Economic Society, MacMillan St Martin's Press.

Knapp G.F. (1973) [1905], The state theory of money, traduction anglaise, New York, M. Kelley Publishers.

Lordon F., Orléan A. (2007), « Genèse de l'État et de la monnaie : le modèle de la potentia multitudinis », Revue du Mauss permanente.

Marx K. (1977) [1867], Le Capital, livre 1, Éditions sociales.

Montalban M. (2012), «De la place de la théorie de la valeur et de la monnaie dans la théorie de la régulation: critique et synthèse $»$, Revue de la régulation, $\mathrm{n}^{\circ} 12,2^{\mathrm{e}}$ semestre.

Patinkin D. (1956), Interest, Money and prices, The MIT Press, 2nd edition.

Salama P. (1992), Introduction à l'économie de Marx, Paris, La Découverte (collection Repères).

Segura A. (1974), «L'inflation est-elle inévitable ? », thèse de doctorat, université de Nice.

Segura A. (1991), "Y-a-t-il une théorie keynésienne implicite de la valeur-travail ?», Économie appliquée, tome XLIV, $\mathrm{n}^{\circ} 3$, p. 131-166.

Segura A. (1995), "Le profit et l'intérêt dans la théorie du circuit », L'actualité économique, vol. 71, $\mathrm{n}^{\circ} 1$, mars, p. 53-70.

Simmel G. (1999), Philosophie de l'argent, Paris, PUF. 